\title{
Habitatbäume im Wirtschaftswald: ökologisches Potenzial und finanzielle Folgen für den Betrieb
}

\author{
Sarah Niedermann-Meier \\ Manuela Mordini \\ Rita Bütler \\ Peter Rotach
}

\author{
Ecolinnea $\mathrm{GmbH}(\mathrm{CH})$ \\ Bern $(\mathrm{CH})$ \\ Eidgenössische Forschungsanstalt WSL und Laboratoire ECOS, EPF Lausanne $(\mathrm{CH})$ \\ Gruppe Waldmanagement/Waldbau, Institut für Terrestrische Ökosysteme, ETH Zürich (CH)*
}

\section{Habitat trees in managed forest: how many are available and to what cost can they be maintained?}

\begin{abstract}
Habitat trees are important elements to maintain forest biodiversity. An effective way to provide habitats for species depending on old growth structures may be the conservation of a certain number of habitat trees integrated in managed forests. It has been suggested to maintain at least five such habitat trees per hectare for this purpose. In order to evaluate the number of existing potential trees as well as the financial consequences for the forest owner, we sampled all trees greater than 40 centimetres at breast height on sample plots of the forest inventory in the forest of Baden, Switzerland. Based on a catalogue of important traits of old trees which are important for the survival of five groups of organisms (insects, birds, bats, lichen, fungi), we assessed the ecological value of all trees. We also estimated their economic value in terms of net wood value for each tree. Results show that the conservation of five currently existing habitat trees per hectare would cause significant financial losses for the owner, amounting to 850 Swiss francs per hectare or 170 francs per tree in our example. Moreover, ecological values of many of these trees would be rather low because there are not enough suitable trees to select from. Ecologically valuable trees most likely were removed during tending of stands. Instead of general demands like for example five trees per hectare it seems more effective to concentrate on the currently existing ecologically valuable trees only. Financial losses may need to be covered by public funding in cases where they are high. Moreover, management of habitat trees needs to be planned on a long-term basis. Conservation and promotion of a certain number of habitat trees need to be integrated in tending concepts from early developmental stages.
\end{abstract}

Keywords: old trees, forest management, ecological assessment, biodiversity, silviculture doi: $10.3188 /$ szf.2010.0391

*Universitätsstrasse 22, CH-8092 Zürich, peter.rotach@env.ethz.ch

M it der Unterzeichnung des internationalen Übereinkommens vom 5. Juni 1992 über die Biologische Vielfalt (SR 0.451.43) hat sich die Schweiz dem Erhalt der biologischen Vielfalt sowie der nachhaltigen Nutzung ihrer Bestandteile verpflichtet. Der Schweizer Wald dient über 40 Prozent aller in der Schweiz lebenden Arten als Lebensraum (Buwal \& WSL 2005). Der Erhalt der Biodiversität in Wäldern ist deshalb im Waldprogramm Schweiz (Projektleitung WAP-CH, BHP Brugger 2004) als eines der prioritären Ziele zur Sicherung einer nachhaltigen Waldbewirtschaftung aufgeführt.

In bewirtschafteten Wäldern besteht vielfach ein Mangel an Alterungsmerkmalen und Naturwaldstrukturen, da Bäume bereits in der Phase ihres wirtschaftlichen Optimums genutzt werden (Möller 2005, Winter et al 2003). Steigende Holzpreise und eine erhöhte Nachfrage nach dem Rohstoff Holz führen dazu, dass diese Tendenz in Zukunft eher zuneh- men wird. Dies könnte sich negativ auf das Vorkommen der xylobionten, das heisst holzbewohnenden, Arten auswirken (Baumgartner 2008). Um diese heute schon vielfach gefährdeten Arten zu erhalten, wurden in der Schweiz bereits verschiedene Massnahmen wie das Ausscheiden von Naturwaldreservaten oder Altholzinseln realisiert (Projektleitung WAP-CH, BHP Brugger 2004). Viele xylobionte Organismen sind jedoch wenig mobil und daher auf die Vernetzung ihrer Lebensgrundlagen angewiesen. Zusätzlich zu Waldreservaten und Altholzinseln ist deshalb eine Integration von Naturschutzmassnahmen im Wirtschaftswald notwendig. Der generelle Mangel an alten und dicken Bäumen mit geeigneten Strukturen wie Totästen, Höhlen, Rissen, Kronenbrüchen oder tiefrissiger Borke lässt sich durch den Erhalt von einzelnen geeigneten Habitatbäumen auf der gesamten Wirtschaftswaldfläche wenigstens teilweise beheben (Winter et al 2003). Habitatbäume 
Für den Waldeigentümer ist der Erhalt von Bäumen zugunsten der Ökologie jedoch mit einer Verminderung der Einnahmen aus der Holzproduktion verbunden. Dies kann in Wirtschaftswäldern zu Interessenkonflikten führen, besonders dort, wo bedeutende ökologische und ökonomische Werte einander gegenüberstehen (Bütler 2005).

Die Forderung nach einer bestimmten Anzahl Habitatbäume pro Hektare Waldfläche wirft zwei wesentliche Fragen auf. Zum einen stellt sich die Frage, ob zum heutigen Zeitpunkt in unseren Wirtschaftswäldern überhaupt genügend geeignete, das heisst ökologisch wertvolle, Bäume vorhanden sind, und zum zweiten, welche finanziellen Auswirkungen für die Waldeigentümer zu erwarten sind, falls eine bestimmte Anzahl solcher Bäume pro Hektare für den Naturschutz aus der Produktion ausscheiden würden.

Zur Beantwortung dieser beiden Fragen wurde im Rahmen von zwei Masterarbeiten an der ETH Zürich ein Modell zur ökologischen Bewertung eines Baumes entwickelt (Meier 2009, Mordini 2009). Mit der Quantifizierung des ökologischen Wertes von Bäumen wird es möglich, diesen Wert zu kommunizieren und zu diskutieren, die vorhandene Anzahl ökologisch wertvoller Bäume zu beziffern und Bäume untereinander zu vergleichen. Es wird überdies möglich, den ökologischen Wert des Baumes mit seinem ökonomischen Wert als Holzprodukt in Beziehung zu setzen und damit mögliche Konflikte zwischen Naturschutz und Holzproduktion finanziell zu beziffern.

Meier (2009) untersuchte die Anzahl und den ökologischen Wert von möglichen, bereits existierenden Habitatbäumen im Wirtschaftswald des Forstbetriebes Baden. Untersucht wurde auch, welche finanziellen Auswirkungen sich für den Betrieb ergeben würden, falls auf die Nutzung unterschiedlicher Mengen dieser Bäume verzichtet wird (Meier 2009).

Eine ökologische und ökonomische Bewertung aller Bäume eines Bestandes ermöglicht ferner, ökologische und wirtschaftliche Veränderungen bei waldbaulichen Eingriffen zu quantifizieren und verschiedene Eingriffsvarianten zu beurteilen. Dadurch lässt sich beantworten, inwieweit sich ökologische und ökonomische Zielsetzungen vereinbaren lassen und auf welche Weise waldbauliche Eingriffe ausgeführt werden müssen, um beide Zielsetzungen möglichst optimal zu vereinen (Mordini 2009).

Neben der Methodik zur ökologischen Bewertung von Bäumen werden in diesem Beitrag hauptsächlich die Ergebnisse der Untersuchungen zur Anzahl vorhandener, möglicher Habitatbäume im Wirtschaftswald von Baden sowie die entstehenden Kosten durch einen Nutzungsverzicht dieser Bäume dargestellt. Die Untersuchung beschränkt sich also darauf, die Kosten zu schätzen, welche dem Betrieb heute entstehen würden, wenn er eine bestimmte 
Anzahl dieser ökologisch wertvollen Bäume heute nicht einschlagen und für immer auf deren Nutzung verzichten würde. Die finanziellen Ergebnisse widerspiegeln folglich nur einen ersten Schritt respektive eine Momentaufnahme. Die Kosten für eine langfristige, nachhaltige Bewirtschaftung von Habitatbäumen wären natürlich deutlich höher, da weitere Kosten entstehen, wenn Habitatbäume anstelle von Z-Bäumen gezielt und nachhaltig ausgelesen und in den jungen Beständen gefördert und erhalten werden sollen. Diese Kosten wurden nicht untersucht und können daher auch nicht beziffert werden. Die Schätzung der langfristigen Kosten ist zudem komplex und spekulativ, da viele Einflussfaktoren (künftige, diskontierte Ein- und Ausgaben, Kalkulationszinssatz, künftige Holzpreise und Sortimente, Abtriebsalter, Ausfallrisiko etc.) zeitlich variieren und daher kaum verlässlich eingeschätzt werden können.

\section{Methode}

\section{Herleitung des ökologischen Modells}

Das Modell zur ökologischen Bewertung eines Baumes basiert auf einem Kriterienkatalog von Strukturmerkmalen, welche für den Erhalt xylobionter Arten von Bedeutung sind, sowie aus einer Kriterienbewertung, mit welcher der ökologische Gesamtwert eines Baumes berechnet wird (Tabelle 1). Für die Kriterien galt die Bedingung, dass sie für im Schweizer Mittelland auftretende Organismen von ökologischer Bedeutung, quantifizierbar sowie im Wald vom Boden her sichtbar beziehungsweise messbar sein müssen.

Für die Auswahl und Bewertung der Kriterien wurden die fünf Organismengruppen Flechten, Fledermäuse, Insekten, Pilze und Vögel berücksichtigt. Gestützt auf Ergebnisse aus der Literatur, auf Expertenbefragungen sowie auf bereits bestehende Ansätze zur Erfassung von ökologisch wertvollen Strukturen an Bäumen (Lachat \& Bütler 2007, Winter et al 2003, Müller 2005, Pro Silva France 2003) wurden die relevanten Kriterien hergeleitet.

Neben 16 Strukturmerkmalen wurden zusätzlich auch der Brusthöhendurchmesser (BHD) sowie die Baumart aufgenommen. Es wurde angenommen, dass der ökologische Wert eines Baumes mit zunehmendem BHD steigt, da er zusätzliche altersspezifische Merkmale und Sonderstrukturen aufweisen kann, die sich nur im Laufe der Zeit entwickeln können (Mulmhöhlen, Rindenstrukturen, starke Totäste etc.). Die Baumarten unterscheiden sich zudem deutlich bezüglich der Zahl der von ihnen abhängigen Arten. Die Bewertung der Baumart erfolgte aufgrund der anhand von Literaturangaben bestimmten Anzahl abhängiger respektive beobachteter Arten je Baumart (vgl. Meier 2009, Mordini 2009).
Anschliessend wurde die Bedeutung jedes Kriteriums durch eine Expertin und vier Experten oder anhand von Literaturrecherchen für jede Organismengruppe sowie für lebende und stehend tote Bäume getrennt mit Punkten bewertet. Für detaillierte Informationen zum Kriterienkatalog sowie zur Kriterienherleitung und -auswahl siehe Meier (2009) und Mordini (2009).

\section{Untersuchungsbetrieb und Datenerhebung}

Der untersuchte Betrieb Baden umfasst rund 700 Hektaren Wald. Da sich Baden an der Nahtstelle zwischen Mittelland und Faltenjura befindet, sind die Bodenverhältnisse äussert vielfältig, was sich im Vorkommen von 38 verschiedenen Waldgesellschaften widerspiegelt. Dominierend sind Buchenwaldgesellschaften auf frischen, tiefgründigen und nährstoffreichen Böden.

Die Datenerfassung erfolgte auf den Probekreisen des bestehenden Stichprobennetzes der Betriebsinventur, welches auf einem Raster von $100 \mathrm{Me}$ ter mal 100 Meter insgesamt 687 Probekreise mit je einer Fläche von drei Aren aufweist. Diese Probekreise sind permanent eingerichtet und im Feld markiert. Da sich die Aufnahmen nur auf den Wirtschaftswald beschränken sollten, reduzierte sich die Anzahl Stichproben durch den Ausschluss der nicht bewirtschafteten Altholzinseln, Reservatsflächen sowie der jungen Lothar-Sturmflächen auf 390 Stichproben. Mit den vereinfachenden Annahmen, dass Bäume erst ab einem BHD von 40 Zentimetern ökologisch wertvoll sind, der BHD im Allgemeinen innert zehn Jahren weniger als zehn Zentimeter zunimmt und Fichten (Picea abies) sowie Tannen (Abies alba) selten ökologisch wertvolle Strukturen aufweisen, konnte die Anzahl aufzunehmender Stichproben aufgrund der Daten der Betriebsinventur von 1997 schliesslich auf 129 Probeflächen reduziert werden. Auf diesen Stichprobenflächen wurden 375 Bäume mit einem BHD von mindestens 40 Zentimetern auf ihren ökologischen und ökonomischen Wert hin untersucht. Um unsere Annahme zu überprüfen, dass junge Bäume mit weniger als 40 Zentimetern BHD weniger ökologisch wertvolle Strukturen aufweisen als dickere Bäume, wurden auf 38 Stichproben auch alle Bäume mit einem BHD von 20 bis 40 Zentimetern taxiert ( $\mathrm{N}=86$ Bäume). Unter der Annahme, dass es sich bei den 129 untersuchten Probeflächen um repräsentative Flächen für den bewirtschafteten Wald handelt, was bei der Betriebsinventur ebenfalls angenommen wird, lassen sich die erhobenen Daten auf die gesamte Fläche des Wirtschaftswaldes hochrechnen.

\section{Erfassung des ökologischen Werts}

Jeder der 461 untersuchten Bäume wurde mithilfe des Kriterienkatalogs (Tabelle 1) angesprochen. Dazu wurde der Baum vom Boden aus von allen Sei- 


\begin{tabular}{|c|c|c|c|c|c|c|}
\hline Kriterien & & $\begin{array}{l}\text { Fleder- } \\
\text { mäuse }\end{array}$ & $\begin{array}{c}\text { Insek- } \\
\text { ten }\end{array}$ & Vögel & $\begin{array}{l}\text { Flech- } \\
\text { ten }\end{array}$ & Pilze \\
\hline \multirow{6}{*}{ Äste } & Tot, ganz oder abgebrochen, Ø: $10-20 \mathrm{~cm}$, Länge: $\geq 50 \mathrm{~cm}$, besonnt & $-/ 1$ & $-/ 4$ & $-/ 2$ & $-/ 3$ & $-/ 2$ \\
\hline & Tot, ganz oder abgebrochen, $\varnothing: 10-20 \mathrm{~cm}$, Länge: $\geq 50 \mathrm{~cm}$, nicht besonnt & $-/ 1$ & -12 & -12 & $-/ 3$ & $-/ 4$ \\
\hline & Tot, ganz oder abgebrochen, Ø: $>20 \mathrm{~cm}$, Länge: $\geq 50 \mathrm{~cm}$, besonnt & $-/ 2$ & $-/ 4$ & $-/ 3$ & $-/ 3$ & $-/ 3$ \\
\hline & Tot, ganz oder abgebrochen, $\varnothing:>20 \mathrm{~cm}$, Länge: $\geq 50 \mathrm{~cm}$, nicht besonnt & $-/ 2$ & $-/ 2$ & -13 & $-/ 3$ & $-/ 4$ \\
\hline & Lebend oder tot, abgebrochen, $\varnothing: \geq 10 \mathrm{~cm}$, Länge $<50 \mathrm{~cm}$ oder ausgefallener Ast & $-/ 2$ & $-/ 2$ & $-/ 1$ & $-/ 3$ & $-/ 2$ \\
\hline & Lebend, abgebrochen, $\varnothing: \geq 10 \mathrm{~cm}$, Länge $\geq 50 \mathrm{~cm}$ & $-/ 2$ & $-/ 3$ & -10 & $-/ 3$ & $-/ 2$ \\
\hline \multirow{8}{*}{$\begin{array}{l}\text { Dürrständer: } \\
\text { zusätzliche } \\
\text { Kriterien }\end{array}$} & Mit Kronenskelett/einzelnen Ästen, Rindenanteil $\geq 50 \%$, Zersetzungsgrad: hart & $4 /-$ & $3 /-$ & $4 /-$ & $4 /-$ & $2 /-$ \\
\hline & Mit Kronenskelett/einzelnen Ästen, Rindenanteil $\geq 50 \%$, Zersetzungsgrad: weich & $3 /-$ & $2 /-$ & $4 /-$ & $4 /-$ & $2 /-$ \\
\hline & Mit Kronenskelett/einzelnen Ästen, Rindenanteil < 50\%, Zersetzungsgrad: hart & $3 /-$ & $3 /-$ & $4 /-$ & $4 /-$ & $3 /-$ \\
\hline & Mit Kronenskelett/einzelnen Ästen, Rindenanteil < 50\%, Zersetzungsgrad: weich & $2 /-$ & $3 /-$ & $4 /-$ & $4 /-$ & $3 /-$ \\
\hline & Stammbruch, Rindenanteil $\geq 50 \%$, Zersetzungsgrad: hart & $3 /-$ & $3 /-$ & $3 /-$ & $4 /-$ & $2 /-$ \\
\hline & Stammbruch, Rindenanteil $\geq 50 \%$, Zersetzungsgrad: weich & $2 /-$ & $3 /-$ & $3 /-$ & $4 /-$ & $2 /-$ \\
\hline & Stammbruch, Rindenanteil < 50\%, Zersetzungsgrad: hart & $2 /-$ & $3 /-$ & $3 /-$ & $4 /-$ & $3 /-$ \\
\hline & Stammbruch, Rindenanteil < 50\%, Zersetzungsgrad: weich & $1 /-$ & $3 /-$ & $3 /-$ & $4 /-$ & $3 /-$ \\
\hline \multirow{4}{*}{ Höhle } & Lochgrösse $(\varnothing):<10 \mathrm{~cm}$, Höhe: $<4 \mathrm{~m}$ & $2 / 2$ & $2 / 1$ & $3 / 3$ & $1 / 1$ & $2 / 2$ \\
\hline & Lochgrösse $(\varnothing):<10 \mathrm{~cm}$, Höhe: $\geq 4 \mathrm{~m}$ & $3 / 3$ & $2 / 1$ & $3 / 3$ & $1 / 1$ & $2 / 2$ \\
\hline & Lochgrösse $(\varnothing): \geq 10 \mathrm{~cm}$, Höhe: $<4 \mathrm{~m}$ & $2 / 2$ & $2 / 2$ & $4 / 4$ & $1 / 1$ & $2 / 2$ \\
\hline & Lochgrösse $(\varnothing): \geq 10 \mathrm{~cm}$, Höhe: $\geq 4 \mathrm{~m}$ & $4 / 4$ & $2 / 2$ & $4 / 4$ & $1 / 1$ & $2 / 2$ \\
\hline \multirow{4}{*}{$\begin{array}{l}\text { Rinden- } \\
\text { schürfung }\end{array}$} & Grösse: $25 \mathrm{~cm}^{2}(5 \mathrm{~cm} \times 5 \mathrm{~cm})$ bis $600 \mathrm{~cm}^{2}(20 \mathrm{~cm} \times 30 \mathrm{~cm})$, Höhe: $<4 \mathrm{~m}$ & $-/ 2$ & $-/ 1$ & $-/ 0$ & $-/ 3$ & $-/ 1$ \\
\hline & Grösse: $25 \mathrm{~cm}^{2}(5 \mathrm{~cm} \times 5 \mathrm{~cm})$ bis $600 \mathrm{~cm}^{2}(20 \mathrm{~cm} \times 30 \mathrm{~cm})$, Höhe: $\geq 4 \mathrm{~m}$ & $-/ 3$ & $-/ 1$ & -10 & -13 & $-/ 2$ \\
\hline & Grösse: $>600 \mathrm{~cm}^{2}(20 \mathrm{~cm} \times 30 \mathrm{~cm})$, Höhe: $<4 \mathrm{~m}$ & $-/ 2$ & $-/ 1$ & $-/ 1$ & $-/ 3$ & $-/ 3$ \\
\hline & Grösse: $>600 \mathrm{~cm}^{2}(20 \mathrm{~cm} \times 30 \mathrm{~cm})$, Höhe: $\geq 4 \mathrm{~m}$ & $-/ 3$ & $-/ 1$ & $-/ 1$ & $-/ 3$ & $-/ 3$ \\
\hline \multirow{2}{*}{$\begin{array}{l}\text { Rinden- } \\
\text { tasche }\end{array}$} & Rindentasche vorhanden, Höhe $<4 \mathrm{~m}$ & $3 / 3$ & $1 / 1$ & $2 / 2$ & $1 / 3$ & $2 / 2$ \\
\hline & Rindentasche vorhanden, Höhe $\geq 4 \mathrm{~m}$ & $4 / 4$ & $1 / 1$ & $2 / 2$ & $1 / 3$ & $2 / 2$ \\
\hline \multirow{8}{*}{$\begin{array}{l}\text { Risse und } \\
\text { Spalten }\end{array}$} & Länge: $5 \mathrm{~cm}$ bis $<100 \mathrm{~cm}$, Höhe: $<4 \mathrm{~m}$, Tiefe: oberflächlich, nicht in Splint & $2 / 2$ & $2 / 2$ & $1 / 1$ & $3 / 3$ & $2 / 2$ \\
\hline & Länge: $5 \mathrm{~cm}$ bis $<100 \mathrm{~cm}$, Höhe: $<4 \mathrm{~m}$, Tiefe: tief, ins Holz hineingehend & $2 / 2$ & $2 / 2$ & $2 / 2$ & $2 / 2$ & $2 / 2$ \\
\hline & Länge: $5 \mathrm{~cm}$ bis $<100 \mathrm{~cm}$, Höhe: $\geq 4 \mathrm{~m}$, Tiefe: oberflächlich, nicht in Splint & $3 / 3$ & $2 / 2$ & $1 / 1$ & $3 / 3$ & $2 / 2$ \\
\hline & Länge: $5 \mathrm{~cm}$ bis $<100 \mathrm{~cm}$, Höhe: $\geq 4 \mathrm{~m}$, Tiefe: tief, ins Holz hineingehend & $3 / 3$ & $2 / 2$ & $2 / 2$ & $2 / 2$ & $2 / 2$ \\
\hline & Länge: $\geq 100 \mathrm{~cm}$, Höhe: $<4 \mathrm{~m}$, Tiefe: oberflächlich, nicht in Splint & $2 / 2$ & $2 / 2$ & $2 / 2$ & $3 / 3$ & $3 / 3$ \\
\hline & Länge: $\geq 100 \mathrm{~cm}$, Höhe: $<4$ m, Tiefe: tief, ins Holz hineingehend & $2 / 2$ & $2 / 2$ & $3 / 3$ & $2 / 2$ & $3 / 3$ \\
\hline & Länge: $\geq 100 \mathrm{~cm}$, Höhe: $\geq 4 \mathrm{~m}$, Tiefe: oberflächlich, nicht in Splint & $3 / 3$ & $2 / 2$ & $2 / 2$ & $3 / 3$ & $3 / 3$ \\
\hline & Länge: $\geq 100 \mathrm{~cm}$, Höhe: $\geq 4 \mathrm{~m}$, Tiefe: tief, ins Holz hineingehend & $3 / 3$ & $2 / 2$ & $3 / 3$ & $2 / 2$ & $3 / 3$ \\
\hline \multirow{11}{*}{$\begin{array}{l}\text { Weitere } \\
\text { Kriterien }\end{array}$} & Moosbewuchs $\geq 25 \%$ & $0 / 0$ & $2 / 2$ & $2 / 2$ & $3 / 3$ & $3 / 2$ \\
\hline & Flechtenbewuchs $\geq 25 \%$ & $0 / 0$ & $2 / 2$ & $2 / 2$ & $4 / 4$ & $3 / 2$ \\
\hline & Efeubewuchs $\geq 25 \%$ & $-2 /-2$ & $1 / 1$ & $3 / 3$ & $-3 /-3$ & $3 / 2$ \\
\hline & Pilzfruchtkörper & $0 / 0$ & $3 / 3$ & $0 / 0$ & $3 / 3$ & $4 / 4$ \\
\hline & Hohlraum am Stammfuss oder Wurzelanlauf & $1 / 1$ & $3 / 3$ & $0 / 0$ & $4 / 4$ & $2 / 1$ \\
\hline & Horst & $0 / 0$ & $0 / 0$ & $3 / 3$ & $2 / 2$ & $1 / 1$ \\
\hline & Hohler Baum, oben geschlossen & $4 / 4$ & $4 / 4$ & $1 / 1$ & $2 / 2$ & $2 / 2$ \\
\hline & Hohler Baum, oben offen & $3 / 3$ & $4 / 4$ & $1 / 1$ & $4 / 4$ & $2 / 2$ \\
\hline & Zwiesel & $2 / 2$ & $0 / 0$ & $0 / 0$ & $3 / 3$ & $2 / 1$ \\
\hline & Stockausschlag & $0 / 0$ & $0 / 0$ & $0 / 0$ & $3 / 3$ & $3 / 1$ \\
\hline & Saftfluss & $0 / 0$ & $3 / 3$ & $2 / 2$ & $3 / 3$ & $2 / 2$ \\
\hline \multirow{4}{*}{ Baumart } & Eiche (Quercus spp.) & $4 / 4$ & $4 / 4$ & $4 / 4$ & $4 / 4$ & $4 / 4$ \\
\hline & Erle (Alnus spp.), Föhre (Pinus sylvestris), Kirschbaum (Prunus spp.), Pappel (Populus spp.) & $2 / 2$ & $2 / 2$ & $2 / 2$ & $2 / 2$ & $2 / 2$ \\
\hline & $\begin{array}{l}\text { Ahorn (Acer spp.), Buche (Fagus sylvatica), Esche (Fraxinus excelsior), Hagebuche } \\
\text { (Carpinus betulus), Lärche (Larix spp.), Linde (Tilia spp.), Ulme (Ulmus spp.) }\end{array}$ & $1 / 1$ & $1 / 1$ & $1 / 1$ & $1 / 1$ & $1 / 1$ \\
\hline & Fichte (Picea abies), Tanne (Abies alba) & $0 / 0$ & $0 / 0$ & $0 / 0$ & $0 / 0$ & $0 / 0$ \\
\hline \multirow{5}{*}{ BHD } & $<40 \mathrm{~cm}$ & $0 / 0$ & $0 / 0$ & $0 / 0$ & $0 / 0$ & $0 / 0$ \\
\hline & $40 \mathrm{~cm}$ bis $<60 \mathrm{~cm}$ & $1 / 1$ & $1 / 1$ & $1 / 1$ & $1 / 1$ & $1 / 1$ \\
\hline & $60 \mathrm{~cm}$ bis $<80 \mathrm{~cm}$ & $2 / 2$ & $2 / 2$ & $2 / 2$ & $2 / 2$ & $2 / 2$ \\
\hline & $80 \mathrm{~cm}$ bis $<100 \mathrm{~cm}$ & $3 / 3$ & $3 / 3$ & $3 / 3$ & $3 / 3$ & $3 / 3$ \\
\hline & $\geq 100 \mathrm{~cm}$ & $4 / 4$ & $4 / 4$ & $4 / 4$ & $4 / 4$ & $4 / 4$ \\
\hline
\end{tabular}

Tab 1 Kriterienkatalog mit Strukturmerkmalen, welche für den Erhalt der fünf berücksichtigten Organismengruppen (Flechten, Fledermäuse, Insekten, Pilze und Vögel) von Bedeutung sind, sowie Kriterienbewertung, mit welcher der ökologische Wert eines Baumes bestimmt werden kann. Die erste Zahl in der Bewertung gilt für tote, die zweite für lebende Bäume. Ein Strich bedeutet, dass das Kriterium nicht aufgenommen wurde. 


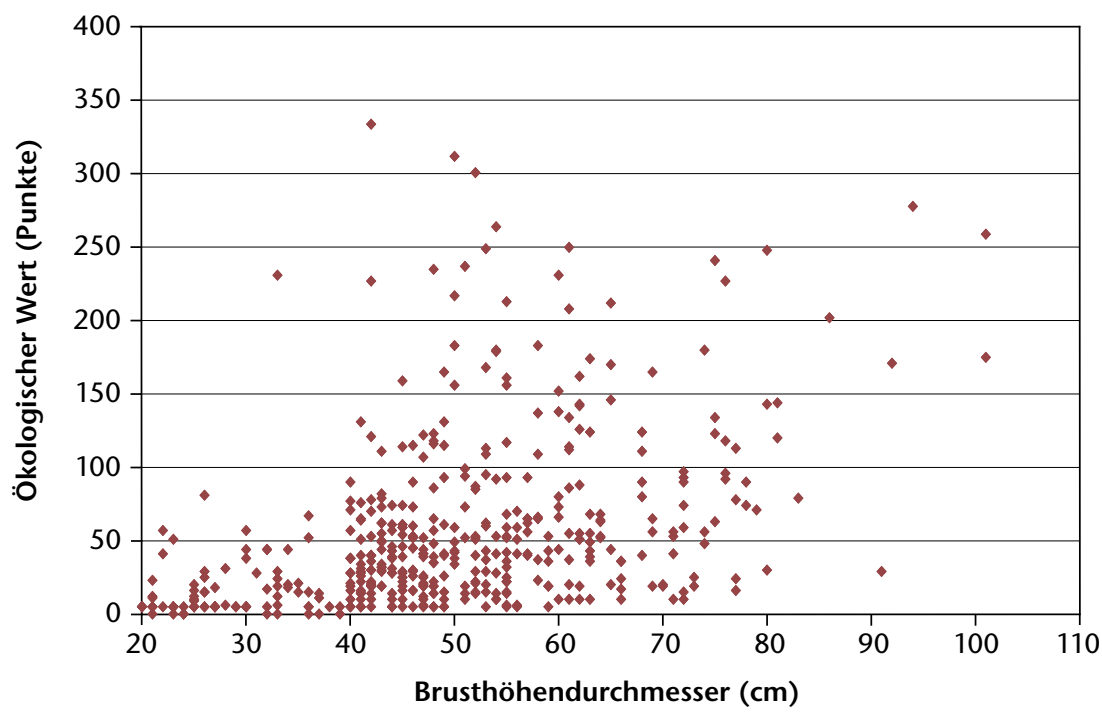

Abb 2 Zusammenhang zwischen ökologischem Wert und dem Brusthöhendurchmesser (BHD) der Bäume.
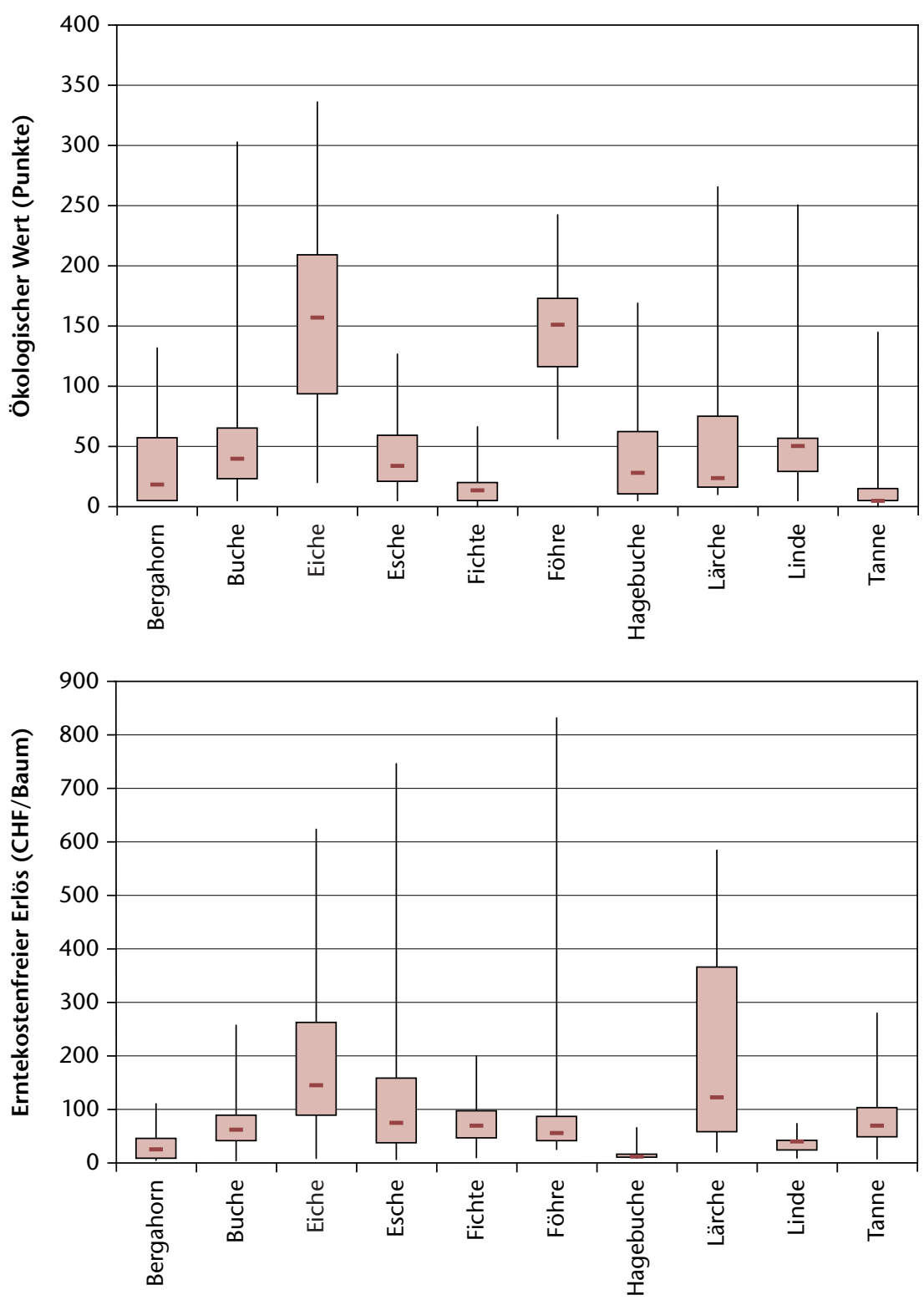

Abb 3 Häufigkeitsverteilung mit Median, Maximum, Minimum, oberem Quartil und unterem Quartil für den ökologischen Wert sowie den erntekostenfreien Erlös der verschiedenen Baumarten (Bäume aller BHD, jedoch ohne Erle, Kirschbaum und Ulme wegen zu geringer Datengrundlage). ten begutachtet, und alle vorhandenen Merkmale wurden festgehalten. Mehrfach an einem Baum vorkommende Strukturen wurden mehrfach erfasst.

Aus Tabelle 1 wird ersichtlich, dass sich die Punkteanzahl pro Organismengruppe in den meisten Kriterien unterscheidet. Bei der ökologischen Bewertung eines Baumes können deshalb sowohl die Anzahl an Strukturmerkmalen als auch die Anzahl der Punkte für ein Kriterium unterschiedlich in die Berechnung miteinbezogen werden. So können die mehrfach an einem Baum vorkommenden Strukturmerkmale nur einfach oder aber mehrfach gewertet werden. Vier Höhlen am gleichen Baum können folglich mit der einfachen Punktesumme oder aber mit der vierfachen Summe in die Bewertung einfliessen. Die Punkte der fünf Organismengruppen können ebenfalls unterschiedlich gewichtet werden. Es ist möglich, nur eine der Organismengruppen zu werten oder die Summe aller Gruppen beziehungsweise deren Mittelwert. Möglich ist ferner, allein den jeweils höchsten Wert der fünf Gruppen zu verwenden, um sicherzustellen, dass die am meisten von dieser Struktur abhängige Gruppe berücksichtigt wird. Diese Berechnungsart wurde beispielsweise von Pro Silva France (2003) gewählt.

Meier (2009) prüfte verschiedene Methoden zur Berechnung des ökologischen Werts eines Baumes. Für die vorliegende Arbeit wurde der ökologische Wert eines Baumes schliesslich als Summe aller Punkte über alle Kriterien und Organismengruppen berechnet. Mehrfach an einem Baum vorkommende Strukturen (beispielsweise mehrere Höhlen) flossen dabei mehrfach in die Berechnung ein.

\section{Erfassung des ökonomischen Werts}

Der ökonomische Wert eines Baumes wurde mit einem modifizierten Computerprogramm von Bont (2005) berechnet. Die Berechnungen basierten hierbei auf einer Sortimentsansprache am stehenden Baum, den aktuellen Holzpreisen (Winter 2008/09), mehreren Parametern zur Berechnung des Holzvolumens (Baumhöhe, BHD, Durchmesser in 7 Metern Höhe, Höhe des Kronenansatzes) sowie den Kosten für die Holzernte. Die Sortiments- und Qualitätsansprache beruhte auf den im Betrieb Baden verwendeten Qualitäten Furnier, B-, C- und D-Qualität (gemäss Handelsgebräuchen für Rundholz) respektive Brennholz. Da gewisse Qualitätsmerkmale (Fäulen, Risse, Farbverkernungen etc.) nur am liegenden Stamm erhoben werden können, handelt es sich bei der Ansprache am stehenden Baum um eine gutachtliche Schätzung des Erlöses. Zur Berechnung der Erntekosten wurden die aktuellen Werte des Betriebes verwendet. Der Gegenwartswert eines Baumes wurde vereinfacht aus dem summierten Holzerlös abzüglich der Erntekosten berechnet (erntekostenfreier Erlös). Bei Bäumen, die noch nicht hiebsreif sind, wurde darauf verzichtet, den künftigen realisierba- 


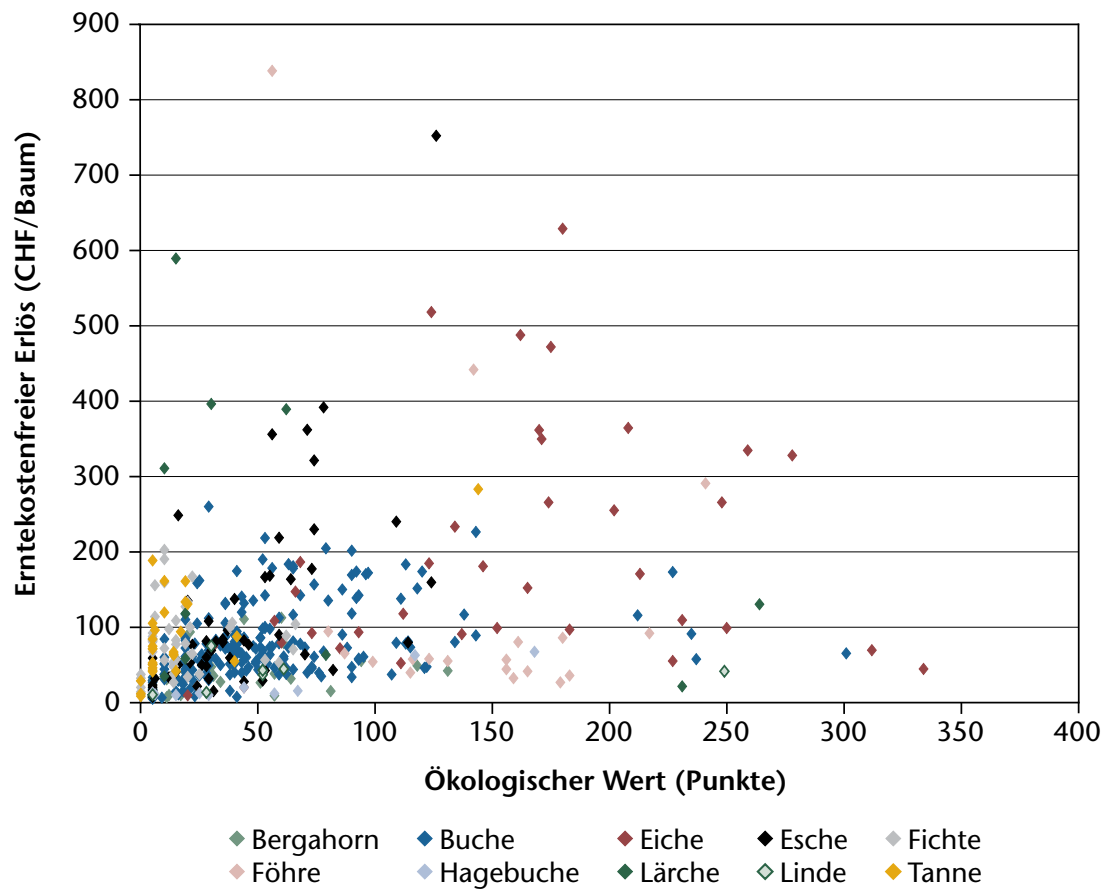

Abb 4 Zusammenhang zwischen ökologischem Wert und erntekostenfreiem Erlös (Erle, Kirschbaum und Ulme wurden aufgrund zu geringer Datengrundlage nicht in die Berechnungen miteinbezogen).

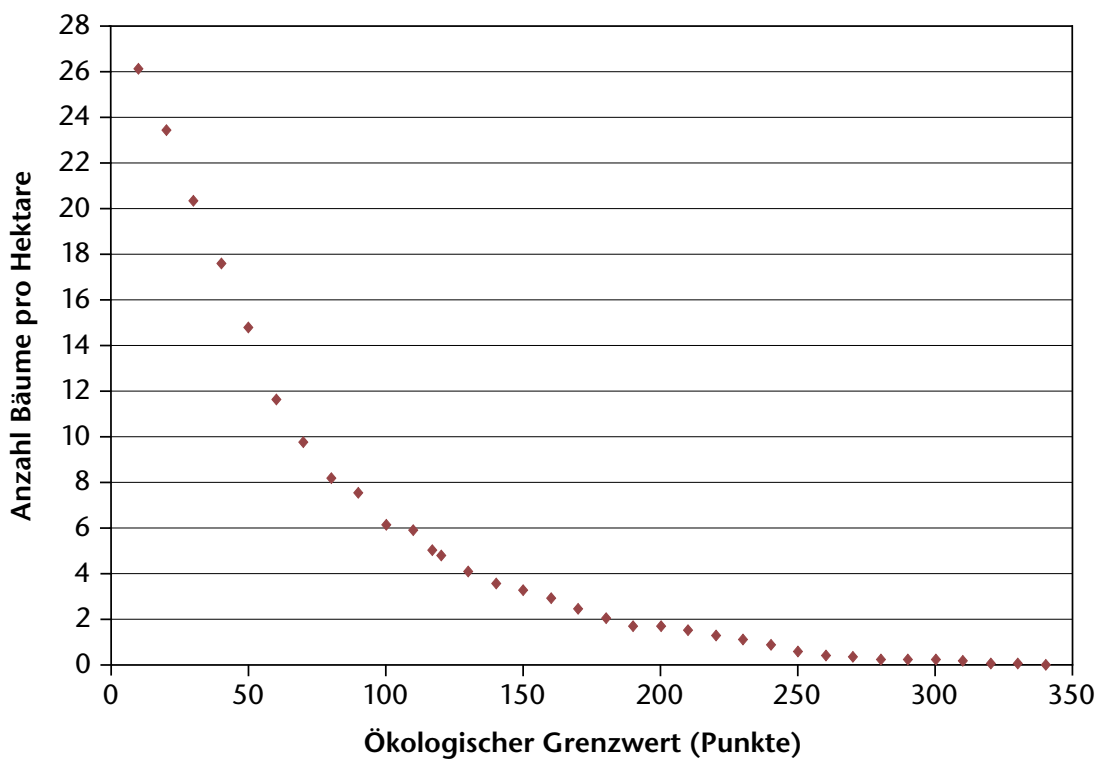

Abb 5 Anzahl vorhandener Bäume pro Hektare Wirtschaftswald bei verschiedenen ökologischen Grenzwerten. lich eine Schätzung. Angesichts des relativ grossen Anteils von Bäumen zwischen 40 und 60 Zentimeter BHD, die ihren Abtriebswert noch nicht erreicht haben, entsprechen die errechneten Werte eher den minimalen Kostenfolgen für den Betrieb.

\section{Resultate}

Die ökologischen Werte der untersuchten Bäume variieren in einem weiten Bereich zwischen 0 und 334 Punkten (Abbildung 2). Mit zunehmendem Durchmesser nimmt der ökologische Wert eines Baumes signifikant $\mathrm{zu}(\mathrm{r}=0.435, \mathrm{p}<0.001)$. Bäume mit einem BHD kleiner als 40 Zentimeter weisen vergleichsweise tiefe ökologische Werte auf. Hingegen können Bäume ab 40 Zentimeter BHD bereits sehr hohe ökologische Werte erreichen.

Die Baumart zeigt einen signifikanten Einfluss auf den ökologischen Wert eines Baumes (Varianzanalyse, $\mathrm{F}[12,448]=27.4 ; \mathrm{p}<0.001$ ) sowie auf seinen erntekostenfreien Erlös ( $\mathrm{F}[12,448]=9.4 ; \mathrm{p}<0.001)$. So erzielt die Eiche sowohl beim ökologischen Wert als auch beim erntekostenfreien Erlös hohe Medianwerte (Abbildung 3). Die Föhre hingegen weist, von Ausnahmen abgesehen, einen vergleichsweise tiefen Erlös auf, erreicht jedoch hohe ökologische Werte, die mit der Eiche vergleichbar sind. Die übrigen Baumarten weisen beim ökologischen Wert deutlich geringere Medianwerte auf, besonders Fichte, Tanne und Bergahorn. Die grossen Streubereiche zeigen allerdings, dass es bei allen Baumarten ausser bei der Fichte auch ökologisch wertvolle Individuen gibt.

Ökologischer und ökonomischer Wert sind signifikant positiv korreliert $(\mathrm{r}=0.343 ; \mathrm{p}<0.001$; $\mathrm{Ab}$ bildung 4). Bei allen Baumarten finden sich allerdings auch Bäume, die einen hohen ökologischen Wert, gleichzeitig aber einen tiefen erntekostenfreien Erlös aufweisen.

Abbildung 5 zeigt, wie viele Bäume ab einem bestimmten ökologischen Wert (= ökologischer Grenzwert) im Wirtschaftswald von Baden zum jetzigen Zeitpunkt vorhanden sind. Unter ökologischem Grenzwert verstehen wir den niedrigsten ökologischen Wert, der akzeptiert werden muss, damit eine bestimmte Anzahl Habitatbäume pro Hektare ausgewählt werden kann. Sollen zum Beispiel fünf Habitatbäume pro Hektare erhalten werden, müssen alle Bäume ab einem ökologischen Grenzwert von 117 oder mehr Punkten berücksichtigt werden. Werden beispielsweise aber nur Bäume mit einem Grenzwert von mehr als 200 Punkten als Habitatbäume akzeptiert, dann finden sich im heutigen Wirtschaftswald von Baden lediglich knapp zwei Stück pro Hektare.

In Abbildung 6 sind die finanziellen Folgen eine Nutzungsverzichtes (ausgedrückt als entgangener erntekostenfreier Erlös) für die gesamte Wirt- 


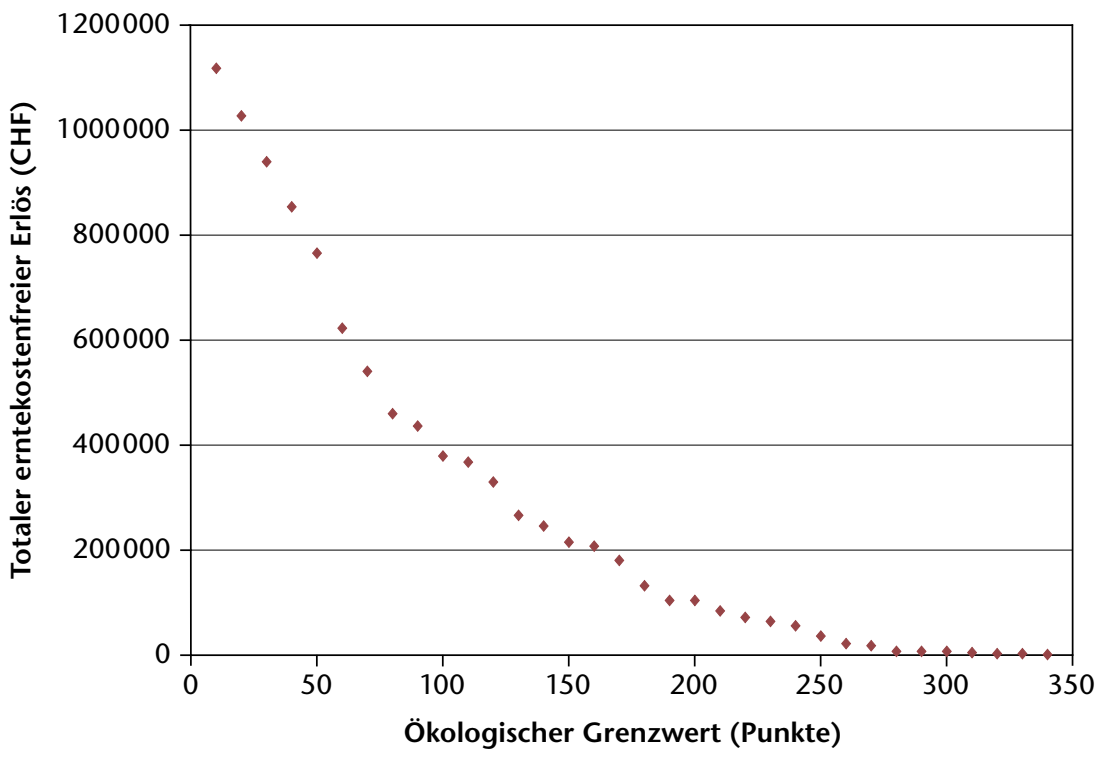

Abb 6 Summierter Verlust (ausgedrückt als entgangener erntekostenfreier Erlös in Schweizer Franken) hochgerechnet auf die gesamte Wirtschaftswaldfläche (390 ha) des Badener Waldes bei verschiedenen ökologischen Grenzwerten.

\begin{tabular}{|c|c|c|c|}
\hline $\begin{array}{c}\text { Anzahl Bäume pro } \\
\text { Hektare }\end{array}$ & $\begin{array}{c}\text { Ökologischer } \\
\text { Grenzwert }\end{array}$ & $\begin{array}{c}\text { Erntekostenfreier } \\
\text { Erlös pro Baum (CHF) }\end{array}$ & $\begin{array}{c}\text { Totaler erntekosten- } \\
\text { freier Erlös (CHF) }\end{array}$ \\
\hline 10 & 67 & 142 & 553800 \\
\hline 9 & 74 & 145 & 512300 \\
\hline 8 & 81 & 145 & 454300 \\
\hline 7 & 91 & 151 & 417800 \\
\hline 6 & 108 & 160 & 378700 \\
\hline 5 & 117 & 172 & 338300 \\
\hline 4 & 132 & 172 & 263000 \\
\hline 3 & 157 & 179 & 208800 \\
\hline 2 & 180 & 164 & 131200 \\
\hline 1 & 232 & 149 & 59600 \\
\hline 0.1 & 313 & 43 & 1400 \\
\hline
\end{tabular}

Tab 2 Ökologische Grenzwerte, mittlere erntekostenfreie Erlöse je Baum und totaler erntekostenfreier Erlös für die Wirtschaftswaldfläche des Forstbetriebes Baden (390 ha; Werte gerundet auf 100 Franken) für verschiedene Dichten von Habitatbäumen je Hektare.

schaftswaldfläche in Abhängigkeit verschiedener ökologischer Grenzwerte dargestellt. Es ist zu erkennen, dass der Forstbetrieb beispielsweise beim Erhalt von fünf Habitatbäumen pro Hektare, das heisst aller Bäume mit einem ökologischen Wert von mindestens 117 Punkten (Abbildung 5), auf knapp 340000 Franken Nettoeinnahmen verzichten müsste.

Tabelle 2 gibt einen Überblick über die ökologischen Grenzwerte, die mittleren erntekostenfreien Erlöse pro Baum sowie die gesamten Verluste (summierte erntekostenfreie Erlöse) für den Betrieb Baden, die beim Erhalt einer bestimmten Menge von Habitatbäumen je Hektare entstehen würden. Es ist zu erkennen, dass ab einer Anzahl von fünf Habitatbäumen pro Hektare und mehr einerseits Bäume mit tiefen ökologischen Werten ausgewählt werden müssten und andererseits sehr hohe Verluste für den Betrieb entstehen würden.

\section{Diskussion}

\section{Bewertung des ökologischen Werts von Bäumen}

Die Untersuchung zeigt, dass verschiedene Kriterien den ökologischen Wert eines Baumes beeinflussen können (Tabelle 1). Mit dem entwickelten Kriterienkatalog und der Kriterienbewertung kann dieser Wert in Punkten ausgedrückt werden. Damit ist es möglich, Bäume hinsichtlich ihres ökologischen Werts zu quantifizieren und miteinander $\mathrm{zu}$ vergleichen. Das ökologische Modell erlaubt ausserdem eine differenzierte Kriterienbewertung, indem eine Beurteilung von Bäumen beispielsweise unter Berücksichtigung lokaler oder regionaler Zielsetzungen vorgenommen werden kann. Wird zum Beispiel die Erhaltung einer Mittelspechtpopulation in einem Gebiet angestrebt, können die für diese Art notwendigen Strukturen respektive Kriterien in die ökologische Bewertung einbezogen werden (Abbildung 7). Ebenso lässt sich die Bewertung der Kriterien spezifisch auf eine Organismengruppe fokussieren.

Die ökologische Ansprache bleibt trotz Kriterienkatalog gutachtlich. Für die Berechnung des ökologischen Wertes sind zudem verschiedene Varianten möglich. Schliesslich stellt unser Modell zur Erhebung des ökologischen Werts von Bäumen aufgrund der komplexen ökologischen Zusammenhänge und Wechselwirkungen lediglich eine Vereinfachung der Realität dar. Mit der Ansprache der Kriterien wird lediglich das Potenzial zur Nutzung eines Kriteriums erfasst, nicht das tatsächliche Vorkommen von Organismen. Letzteres hängt nicht nur vom Vorhandensein der Strukturen ab, sondern auch von Faktoren wie der Anzahl geeigneter Habitate in der Umgebung, der Verbreitungsmöglichkeit, den Flächenansprüchen und so weiter (für Details sowie eine ausführliche Diskussion der ökologischen Bewertung siehe Meier [2009] und Mordini [2009]).

\section{Anzahl und Kosten von Habitatbäumen im Betrieb Baden}

Ökologischer und ökonomischer Wert zeigen eine positive Korrelation, was auf eine tendenzielle Konfliktsituation zwischen Naturschutz und Holzproduktion hindeutet, vor allem bei jenen Bäumen, die sowohl hohe ökologische wie hohe ökonomische Werte aufweisen. Dies betrifft zumeist Eichen. Da die Verteilung der ökonomischen wie der ökologischen Werte jedoch stark streut (Abbildung 4), finden sich aber auch Bäume, die ohne grosse Konflikte respektive Kostenfolgen erhalten werden können. Ideal sind Bäume, die einen hohen ökologischen und gleichzeitig einen tiefen ökonomischen Wert aufweisen. Diese Bäume sollten besondere Beachtung finden und unbedingt erhalten bleiben. Die Resultate zeigen, dass ökologisch wertvolle Bäume mit geringem ökonomischem Wert zwar vorkommen, aber 
zept umgesetzt, welches die gezielte Erhaltung der ökologisch wertvollen Habitatbäume und Habitatbaumgruppen auf der gesamten Wirtschaftswaldfläche beinhaltet und durch einen Partner aus der Privatwirtschaft im Rahmen eines Ökosponsorings finanziell unterstützt wird. Zudem wird das Forstpersonal gezielt sensibilisiert und geschult.

Eine Möglichkeit zur gezielten Schulung von Forstpersonen stellt das Marteloskop dar. Ein Marteloskop ist eine abgegrenzte Fläche, auf welcher jeder Baum ab einem bestimmten Brusthöhendurchmesser (BHD) kartiert, nummeriert und durch verschiedene Parameter charakterisiert ist. Darin können waldbauliche Eingriffe simuliert und per Computerprogramm ausgewertet werden. Ein Marteloskop kann neben der Verwendung zu Schulungszwecken auch einen Beitrag zum fachlichen Austausch von Forstpersonen (Bruciamacchie 2006) sowie zum Dialog zwischen verschiedenen Akteuren in der Waldbewirtschaftung leisten, indem es Eingriffe sichtbar und diskutierbar macht (Gauquelin et al 2008). In Tägerwilen, Kanton Thurgau, wurden zwei solcher Marteloskope eingerichtet (Mordini 2009).

Kasten Was ist ein Marteloskop?

\section{Schlussfolgerungen}

Die Erhaltung von Habitatbäumen ist eine Aufgabe, die im multifunktionalen Wirtschaftswald Platz haben muss. Wir haben eine Methode entwickelt, um den ökologischen Wert von Habitatbäumen möglichst objektiv zu erfassen und mit dem ökonomischen Wert zu vergleichen. Die Ergebnisse aus dem Untersuchungsgebiet zeigen, dass der Nutzungsverzicht bereits von wenigen Habitatbäumen mit einer relativ grossen finanziellen Einbusse verbunden ist. Man kann sich deshalb fragen, ob die Forderung für den Erhalt von Habitatbäumen nicht über den Grundauftrag des naturnahen Waldbaus hinausgeht und ohne Entschädigung für die Waldeigentümer tatsächlich erwartet werden kann. Besser als eine pauschale Forderung für eine bestimmte Anzahl Habitatbäume pro Hektare eignen sich gemäss unseren Ergebnissen flexible Konzepte, die sich auf die vorhandenen, ökologisch wertvollen Bäume konzentrieren, deren Erhalt finanziell entschädigen und für die langfristige Förderung zukünftiger Habitatbäume im Wirtschaftswald sorgen. Für eine langfristige, nachhaltige Förderung von Habitatbäumen sind weitere finanzielle Einbussen unvermeidlich.

Eingereicht: 26. März 2010, akzeptiert (mit Review): 8. Juni 2010

\section{Dank}

Wir danken Beatrice Senn-Irlet, Simon Birrer, Martin Obrist, Christoph Scheidegger und Beat Wermelinger für ihre fachliche Unterstützung als Expertin/Experten. Unser Dank gilt ferner dem Stadtforstamt Baden, insbesondere dem Stadtoberförster Georg
Schoop, sowie der Bürgergemeinde Tägerwilen, Hans Imper, Gerold Schwager und Erich Tiefenbacher für die fachliche Unterstützung und für das Zurverfügungstellen der zwei Untersuchungsflächen. Ausserdem danken wir Leo Bont im Zusammenhang mit der Weiterentwicklung seines Computerprogramms.

\section{Literatur}

BAUMGARTNER H (2008) Naturnaher Waldbau: Holzen im multifunktionalen Wald. Bern: Bundesamt Umwelt, Magazin Umwelt (4), pp. 15-20.

BONFILS P, HORISBERGER D, ULBER M, EDITORS (2005) Förderung der Eiche. Strategie zur Erhaltung eines Natur- und Kulturerbes der Schweiz. Bern: Bundesamt Umwelt Wald Landschaft, Schriftenreihe Umwelt 383. 102 p.

BONT L (2005) Kundenorientierte Optimierung der Sortiments-Aushaltung bei der Holzernte. Zürich: ETH Zürich, Diplomarbeit. 205 p.

BRUCIAMACCHIE M (2006) Le marteloscope, un outil pour apprendre la gestion durable - exemple d'évaluation de différents scénarios au marteloscope de Zittersheim (Vosges du Nord). In: Valauri D, André J, Dodelin B, Eynard-Machet $R$, Rambaud $D$, editors. Bois mort et à cavités - une clé pour des forêts vivantes. Paris: Lavoisier. pp. 237-252.

BÜTLER R, LACHAT T, SCHLAEPFER R (2006) Saproxylische Arten in der Schweiz: ökologisches Potenzial und Hotspots. Schweiz Z Forstwes 157: 208-216. doi: 10.3188/ szf.2006.0208

BÜTLER R (2005) Alt- und Totholz. Ein Zeichen moderner, nachhaltiger Waldwirtschaft. Wald Holz 86 (4): 45-48.

BUWAL, WSL (2005) Waldbericht 2005 - Zahlen und Fakten zum Zustand des Schweizer Waldes. Bern: Bundesamt Umwelt Wald Landschaft. $151 \mathrm{p}$.

GAUQUELIN X, COURBAUD B, FAY J, BERGER F, MERMIN E (2008) Conduite de peuplements mélangés en forêts de montagne: exemple d'une collaboration chercheurs-gestionnaires. Revue For Fr 60 (2): 207-214.

HAHN P, HEYNEN D, INDERMÜHLE M, MOLLET P, BIRRER S (2005) Holznutzung und Naturschutz. Praxishilfe mit waldbaulichen Merkblättern. Bundesamt Umwelt Wald Landschaft, Vollzug Umwelt. 113 p.

LACHAT T, BÜTLER R (2007) Gestion des vieux arbres et du bois mort - îlots de sénescence, arbres-habitat et métapopulations saproxyliques. www.wsl.ch/forschung/forschungsunits/walddynamik/diversitaet/totholzmanagement/rapport_bafu_2007.pdf (12.6.10).

MEIER S (2009) Beurteilung des ökologischen und ökonomischen Wertes von Habitatbäumen in Wirtschaftswäldern am Beispiel des Forstbetriebs Baden. Zürich: ETH Zürich, Masterarbeit. $107 \mathrm{p}$.

MORDINI M (2009) Modellierung und Beurteilung der ökologischen und ökonomischen Wirkungen von waldbaulichen Eingriffen - Einrichtung zweier Marteloskope in eichenreichen Flächen. Zürich: ETH Zürich, Masterarbeit. 118 p.

MÖLLER G (2005) Habitatstrukturen holzbewohnender Insekten und Pilze. Recklinghausen: Landesanstalt Ökologie Bodenordnung Forsten, Mitt 3/2005. pp. 30-35.

MÜLLER J (2005) Waldstrukturen als Steuergrösse für Artengemeinschaften in kollinen bis submontanen Buchenwäldern. Weihenstephan: TU München. PhD-thesis. deposit. ddb.de/cgi-bin/dokserv?idn=978997042 (11.6.2010). 
PROJEKTLEITUNG WAP-CH, BHP BRUGGER (2004) Waldprogramm Schweiz (WAP-CH). Bern: Bundesamt Umwelt Wald Landschaft, Schriftenreihe Umwelt 363. 119 p.

PRO SILVA FRANCE (2003) Méthodologie d'évaluation du coût d'un martelage favorable à certains éléments de la biodiversité - cas de la forêt de Zittersheim. www.prosilva.fr/ brochures/brochure_EtudePSF-PNR.pdf (28.6.2010).

\section{Habitatbäume im Wirtschaftswald: ökologisches Potenzial und finanzielle Folgen für den Betrieb}

Die Erhaltung der Biodiversität im Wald ist eine wichtige Aufgabe des multifunktionalen Waldmanagements. Für die Erhaltung von Arten, die auf Strukturen reifer Waldphasen angewiesen sind, besteht eine wirksame Möglichkeit darin, eine bestimmte Anzahl an Habitatbäumen im bewirtschafteten Wald zu erhalten. Gefordert werden im Allgemeinen mindestens fünf Habitatbäume pro Hektare.

Um zu untersuchen, wie viele potenzielle Habitatbäume gegenwärtig vorhanden sind und welche finanziellen Folgen die Erhaltung einer bestimmten Anzahl dieser Bäume für den Waldeigentümer bedeuten würde, wurden im Forstbetrieb der Stadt Baden alle Bäume mit einem BHD von mehr als $40 \mathrm{~cm}$ auf allen Stichprobenflächen der Betriebsinventur erfasst. Basierend auf einem Katalog von wichtigen Strukturmerkmalen, die für Vorkommen und Überleben von fünf Gruppen von Organismen (Insekten, Vögel, Fledermäuse, Flechten und Pilze) entscheidend sind, wurden diese Bäume ökologisch bewertet. Für die ökonomische Bewertung wurde der erntekostenfreie Erlös ermittelt.

Die Ergebnisse zeigen, dass ein Nutzungsverzicht von fünf Habitatbäumen pro Hektare bedeutende finanzielle Folgen von bis zu CHF 850.- je Hektare respektive CHF 170.- je Habitatbaum oder CHF 340000 .- für die gesamte Wirtschaftswaldfläche des Forstbetriebes Baden nach sich zieht. Dabei ist der ökologische Wert vieler dieser Bäume eher tief, weil nicht genügend Bäume zur Auswahl vorhanden sind. Die ökologisch wertvollen Bäume werden offensichtlich bereits im Laufe der Bestandespflege zugunsten ökonomisch wertvoller Bäume entnommen.

Konzepte mit einer fixen Anzahl an Habitatbäumen je Hektare scheinen aufgrund unserer Ergebnisse wenig wirksam und finanziell kostspielig. Zweckmässiger erscheint eine Konzentration auf die ökologisch wirklich wertvollen Bäume. Unsere Ergebnisse zeigen zudem, dass eine finanzielle Abgeltung des Waldeigentümers für das Belassen von Habitatbäumen notwendig ist, da die finanziellen Verluste derart gross sind, dass sie nicht im Rahmen des «naturnahen Waldbaus» erwartet werden können. Das Management von Habitatbäumen muss zudem langfristig geplant werden. Die Erhaltung und Förderung einer bestimmten Anzahl solcher Bäume muss bereits in den Pflegekonzepten berücksichtigt werden.
WINTER S, SCHUMACHER H, FLADE M, MÖLLER G (2003) Naturschutzstandards für die Bewirtschaftung von Buchenwäldern im nordostdeutschen Tiefland. Eberswalde: Landesanstalt für Grossschutzgebiete, Sachbericht über das F\&E-Vorhaben «Biologische Vielfalt und Forstwirtschaft». $445 \mathrm{p}$.

\section{Arbres-habitat en forêt de production: Combien y en a-t-il et quel est le coût de leur maintien?}

Le maintien de la biodiversité en forêt est une tâche prioritaire de la gestion forestière multifonctionnelle. Une façon efficace de favoriser les espèces dépendantes des structures de sénescence des vieux peuplements est le maintien d'un certain nombre d'arbres-habitat en forêt de production. Les recommandations à ce sujet préconisent en général de garder cinq arbres-habitat par hectare.

Notre objectif était de quantifier le nombre d'arbres-habitat potentiels, ainsi que d'estimer le coût résultant du maintien d'un certain nombre de ces arbres. Dans les forêts publiques de la ville de Baden, nous avons attribué une taxation écologique et économique à tous les arbres d'un DHP supérieur à $40 \mathrm{~cm}$ dans les placettes de l'inventaire forestier. Chaque arbre a reçu une note écologique sur la base d'un catalogue de structures d'habitat qui sont importantes pour la présence de cinq groupes d'organismes (insectes, oiseaux, chauvessouris, lichens et champignons). De plus, nous avons calculé les recettes nettes de chaque arbre.

Les résultats montrent que les conséquences financières dues au maintien présumé de cinq arbres-habitat par hectare sont importantes et se montent à CHF 850.- par hectare respectivement à CHF 170.- par arbre-habitat ou un total de CHF 340000 .- pour l'ensemble de la surface de la forêt de production de l'entreprise. La valeur écologique des ces arbres est relativement faible car il n'y a pas assez d'arbres à sélectionner. Apparemment, les arbres-habitat présentant un grand potentiel écologique sont éliminés lors des éclaircies en faveur d'arbres à haute valeur économique.

Nos résultats montrent que les concepts exigeant un nombre fixe d'arbres-habitat par hectare ne sont pas forcément efficaces et, de plus, peuvent s'avérer coûteux. II nous semble plus pertinent de se concentrer sur les arbres-habitat à haute valeur écologique. Nos résultats laissent penser qu'il est nécessaire d'indemniser le propriétaire forestier pour le maintien d'arbres-habitat, au vue de la perte financière importante qui dépasse ce que la «sylviculture proche de la nature» peut raisonnablement exiger. En plus, la gestion des arbres-habitat doit être planifiée à long terme. Il est nécessaire d'intégrer le maintien d'un certain nombre d'arbres-habitat dans les concepts de soins aux peuplements déjà. 